\title{
THE TIME TO ABSORPTION IN $\Lambda$-COALESCENTS
}

\author{
BY GÖTZ KERSTING AND ANTON WAKOLBINGER
}

\begin{abstract}
We present a law of large numbers and a central limit theorem for the time to absorption of $\Lambda$-coalescents with dust started from $n$ blocks, as $n \rightarrow \infty$. The proofs rely on an approximation of the logarithm of the block-counting process by means of a drifted subordinator.

Keywords: Coalescent; time to absorption; law of large numbers; central limit theorem; subordinator with drift

2010 Mathematics Subject Classification: Primary 60J75

Secondary 60J27; 60F05
\end{abstract}

\section{Introduction and main results}

Given a large sample of individuals with a common ancestor, how long are the ancestral lineages back to that ancestor? For a population of constant size, this question concerns the absorption time of a coalescent which describes the genealogical tree of $n$ individuals by means of merging partitions. Here we consider coalescents with multiple mergers, also known as $\Lambda$-coalescents, as introduced in 1999 by Pitman [6] and Sagitov [7]. If $\Lambda$ is a finite, nonzero measure on $[0,1]$, then the $\Lambda$-coalescent started with $n$ blocks is a continuous-time Markov chain $\left(\Pi_{n}(t), t \geq 0\right)$ taking its values in the set of partitions of $\{1, \ldots, n\}$. It has the property that whenever there are $b$ blocks, each possible transition that involves merging $k \geq 2$ of the blocks into a single block happens at rate

$$
\lambda_{b, k}=\int_{[0,1]} p^{k}(1-p)^{b-k} \frac{\Lambda(\mathrm{d} p)}{p^{2}},
$$

and these are the only possible transitions. Let $N_{n}(t)$ be the number of blocks in the partition $\Pi_{n}(t), t \geq 0$. Then

$$
\tau_{n}:=\inf \left\{t \geq 0: N_{n}(t)=1\right\}
$$

is the time of the last merger, also called the absorption time of the coalescent started in $n$ blocks. In this paper we study the asymptotic distribution of $\tau_{n}$ as $n \rightarrow \infty$.

Our first result is a law of large numbers for the times $\tau_{n}$. Let

$$
\mu:=\int_{[0,1]}|\log (1-p)| \frac{\Lambda(\mathrm{d} p)}{p^{2}}
$$

in particular, $\mu=\infty$ when $\Lambda(\{0\})>0$ or $\Lambda(\{1\})>0$.

Theorem 1. For any $\Lambda$-coalescent, as $n \rightarrow \infty$,

$$
\frac{\tau_{n}}{\log n} \rightarrow \frac{1}{\mu} \quad \text { in probability. }
$$

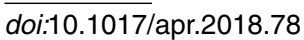

(C) Applied Probability Trust 2018 
This theorem says that in a $\Lambda$-coalescent the number of blocks decays at least at an exponential rate. If $\mu=\infty$ then the right-hand limit is 0 , and the coalescent decreases even super-exponentially fast. The $\mu<\infty$ case is equivalently captured by the simultaneous validity of the two conditions

$$
\int_{[0,1]} \frac{\Lambda(\mathrm{d} p)}{p}<\infty \text { and } \int_{[0,1]}|\log (1-p)| \Lambda(\mathrm{d} p)<\infty .
$$

The first condition is a requirement on $\Lambda$ in the neighbourhood of 0 : it prohibits a swarm of small mergers (these can occur in coalescents coming down from $\infty$, meaning that the $\tau_{n}$ are bounded in probability uniformly in $n$ ). The second is a condition on $\Lambda$ in the vicinity of 1: it rules out the possibility of mergers which, although appearing only occasionally, are so vast that they make the coalescent collapse. Herriger and Möhle [3] obtained a counterpart to Theorem 1 in which $\tau_{n}$ in (1) is replaced by its expectation.

Our second result is a central limit theorem. Here we confine ourselves to coalescents with $\mu<\infty$. Then the function

$$
f(y):=\int_{[0,1]} \frac{1-(1-p)^{\mathrm{e}^{y}}}{\mathrm{e}^{y}} \frac{\Lambda(\mathrm{d} p)}{p^{2}}, \quad y \in \mathbb{R},
$$

is everywhere finite. Also, $f$ is a positive, monotone decreasing, continuous function with the property $f(y) \rightarrow 0$ for $y \rightarrow \infty$. Let

$$
b_{n}:=\int_{\kappa}^{\log n} \frac{\mathrm{d} y}{\mu-f(y)},
$$

where we choose $\kappa \geq 0$ such that

$$
f(y) \leq \frac{1}{2} \mu \text { for all } y \geq \kappa .
$$

Theorem 2. Assume that $\mu<\infty$ and, moreover,

$$
\sigma^{2}:=\int_{[0,1]}(\log (1-p))^{2} \frac{\Lambda(\mathrm{d} p)}{p^{2}}<\infty .
$$

Then, as $n \rightarrow \infty$,

$$
\frac{\tau_{n}-b_{n}}{\sqrt{\log n}} \stackrel{\mathrm{D}}{\rightarrow} N\left(0, \frac{\sigma^{2}}{\mu^{3}}\right) .
$$

Under the additional condition

$$
\int_{[0,1]}|\log p| \frac{\Lambda(\mathrm{d} p)}{p}<\infty
$$

Gnedin et al. [1] obtained the CLT (3) with $b_{n}$ replaced by $(\log n) / \mu$ (condition (9) in [1] is equivalent to the condition at (4); see [4, Remark 13]). Thus, the question arises, whether the simplified centering by $(\log n) / \mu$ is always feasible. The next proposition shows that this can be done under a condition that is weaker than (4), but not in every case. 
Proposition 1. Let $0 \leq c<\infty$. Then

$$
b_{n}=\frac{\log n}{\mu}+\frac{2 c}{\mu^{2}} \sqrt{\log n}+o(\sqrt{\log n}) \text { as } n \rightarrow \infty
$$

if and only if

$$
\sqrt{|\log r|} \int_{[0, r]} \frac{\Lambda(\mathrm{d} p)}{p} \rightarrow c \quad \text { as } r \rightarrow 0 .
$$

Example. Consider for $\gamma \in \mathbb{R}$ the finite measures

$$
\Lambda(\mathrm{d} p)=\left(1+\log \frac{1}{p}\right)^{-\gamma} \mathrm{d} p, \quad 0 \leq p \leq 1 .
$$

For $\gamma=0$, this gives the Bolthausen-Sznitman coalescent. For $\gamma>1$, it yields coalescents with $\mu, \sigma^{2}<\infty$. Note that (4) is satisfied if and only if $\gamma>2$, and (6) is fulfilled if and only if $\gamma>\frac{3}{2}$. Thus, within the range $1<\gamma \leq \frac{3}{2}$, we have to come back to the constants $b_{n}$ in the central limit theorem.

The law of large numbers from Theorem 1 holds for all $\gamma>1$. For the regime $\gamma \leq 1$, Theorem 1 tells us only that $\tau_{n}=o_{P}(\log n)$. For $\gamma=0$, the Bolthausen-Sznitman coalescent, it is known that $\tau_{n}$ is already down to the order $\log \log n$ [2]. For $\gamma<0$, applying Schweinsberg's criterion [8], it can be shown that the coalescents come down from $\infty$. There remains the gap $0<\gamma \leq 1$. It is tempting to conjecture that $\tau_{n}$ is of order $(\log n)^{\gamma}$ for $0<\gamma<1$.

When equation (6) does not hold, then the approximation to $b_{n}$ that follows may be practical. Starting from the identity

$$
\frac{1}{\mu-f(y)}=\frac{1}{\mu}+\frac{f(y)}{\mu^{2}}+\frac{f^{2}(y)}{\mu^{3}}+\cdots+\frac{f^{k}(y)}{\mu^{k+1}}+\frac{f^{k+1}(y)}{\mu^{k+1}(\mu-f(y))},
$$

we obtain the expansion

$$
b_{n}=\frac{\log n}{\mu}+\frac{1}{\mu^{2}} \int_{0}^{\log n} f(y) \mathrm{d} y+\cdots+\frac{1}{\mu^{k+1}} \int_{0}^{\log n} f^{k}(y) \mathrm{d} y+O\left(\int_{0}^{\log n} f^{k+1}(y) \mathrm{d} y\right) .
$$

We now explain the method of proving Theorems 1 and 2. We are dealing mainly with $\Lambda$-coalescents that have a dust component. Briefly speaking, these are the coalescents for which the rate at which a single lineage merges with some others from the sample remains bounded as the sample size tends to $\infty$. It is well known (see, e.g. [6, Theorem 8]) that this property is characterized by the condition

$$
\int_{[0,1]} \frac{\Lambda(\mathrm{d} p)}{p}<\infty
$$

An established tool for the analysis of a $\Lambda$-coalescent with dust is the subordinator $S=\left(S_{t}\right)_{t \geq 0}$; this is used to approximate the logarithm of its block-counting process $N_{n}=\left(N_{n}(t)\right)_{t \geq 0}$ (see, e.g. [1], [5], and [6]). We recall this subordinator in Section 3. Indeed, analogues of Theorems 1 and 2 are well known for first-passage times of subordinators with finite first and second moments, respectively, but this approximation neglects the subtlety that a coalescent of $b$ lineages results in a downward jump of size $b-1$ (and not $b$ ) for the process $N_{n}$. This effect 
becomes significant when many small jumps accumulate over time, as happens close to the dustless case (this is readily seen in Proposition 1 and the above example). Then the appropriate approximation is provided by a drifted subordinator $Y_{n}=\left(Y_{n}(t)\right)_{t \geq 0}$, given by the stochastic differential equation

$$
Y_{n}(t)=\log n-S_{t}+\int_{0}^{t} f\left(Y_{n}(s)\right) \mathrm{d} s, \quad t \geq 0,
$$

with initial value $Y_{n}(0)=\log n$. The drift compensates the difference between $b$ and $b-1$ just mentioned. In Kersting et al. [4] it was shown that

$$
\sup _{t<\tau_{n}}\left|Y_{n}(t)-\log N_{n}(t)\right|=O_{P}(1) \quad \text { as } n \rightarrow \infty
$$

that is, these random variables are bounded in probability. In Section 3 we suitably strengthen this result. In Section 2 we provide the required limit theorems for passage times for a more general class of drifted subordinators. The above results are then proved in Section 4.

It turns out that the regime considered by Gnedin et al. [1] is one in which the random variables $\int_{0}^{\tau_{n}} f\left(Y_{n}(s)\right) \mathrm{d} s$ are bounded in probability uniformly in $n$. This can be seen to be equivalent to the requirement $\int_{0}^{\infty} f(y) \mathrm{d} y<\infty$, which is likewise equivalent to (4) (see the proof of [4, Corollary 12]). Under this assumption, Gnedin et al. [1] proved their central limit theorem also with nonnormal (stable or Mittag-Leffler) limiting distributions for $\tau_{n}$. A similar generalization of Theorem 2 is feasible in the general dust case, without requirement (4).

\section{Limit theorems for a drifted subordinator}

Let $S=\left(S_{t}\right)_{t \geq 0}$ be a pure-jump subordinator with Lévy measure $\lambda$ on $(0, \infty)$. Recall that this requires

$$
\int_{0}^{\infty}(y \wedge 1) \lambda(\mathrm{d} y)<\infty .
$$

More generally than the specific function in (2), let $f: \mathbb{R} \rightarrow \mathbb{R}$ be an arbitrary positive, nonincreasing, continuous function with

$$
\lim _{y \rightarrow \infty} f(y)=0 .
$$

Let the process $Y^{z}=\left(Y_{t}^{z}\right)_{t \geq 0}$ denote the unique solution of the stochastic differential equation

$$
Y_{t}^{z}=z-S_{t}+\int_{0}^{t} f\left(Y_{s}^{z}\right) \mathrm{d} s
$$

with initial value $z>0$; we investigate the asymptotic behaviour in the limit $z \rightarrow \infty$ of its passage times across $x \in \mathbb{R}$, that is, of

$$
T_{x}^{z}:=\inf \left\{t \geq 0: Y_{t}^{z}<x\right\}
$$

Our first result provides a law of large numbers. Denote

$$
\mu:=\int_{(0, \infty)} y \lambda(\mathrm{d} y)
$$


Proposition 2. Assume that $\mu<\infty$. Then, for any $x \in \mathbb{R}$, as $z \rightarrow \infty$,

$$
\frac{1}{z} T_{x}^{z} \rightarrow \frac{1}{\mu} \text { in probability. }
$$

Proof. Let $z>x$. Then

$$
\left\{T_{x}^{z} \geq t\right\}=\left\{Y_{s}^{z} \geq x \text { for all } s \leq t\right\}=\left\{S_{s} \leq z-x+\int_{0}^{s} f\left(Y_{u}^{z}\right) \mathrm{d} u \text { for all } s \leq t\right\} .
$$

The positivity of $f$ implies that $\mathbb{P}\left(T_{x}^{z} \geq t\right) \geq \mathbb{P}\left(S_{t} \leq z-x\right)$, so, for any $\varepsilon>0$,

$$
\mathbb{P}\left(T_{x}^{z} \geq(1-\varepsilon) \frac{z}{\mu}\right) \geq \mathbb{P}\left(S_{(1-\varepsilon) z / \mu} \leq z-x\right) .
$$

Now $\mu=\mathbb{E}\left[S_{1}\right]$, so, by the law of large numbers,

$$
\lim _{t \rightarrow \infty} \frac{S_{t}}{t}=\mu \quad \text { a.s.; }
$$

hence, the right-hand term in (10) converges to 1 as $z \rightarrow \infty$ and also

$$
\mathbb{P}\left(T_{x}^{z} \geq(1-\varepsilon) \frac{z}{\mu}\right) \rightarrow 1 .
$$

On the other hand, $\left\{T_{x}^{z} \geq t\right\}$ equals

$$
\left\{Y_{s}^{z} \geq x \text { for all } s \leq t\right\}=\left\{Y_{s}^{z} \geq x \text { for all } s \leq t, S_{t} \leq z-x+\int_{0}^{t} f\left(Y_{s}^{z}\right) \mathrm{d} s\right\} .
$$

Monotonicity of $f$ implies that $\mathbb{P}\left(T_{x}^{z} \geq t\right) \leq \mathbb{P}\left(S_{t} \leq z-x+t f(x)\right)$. Therefore, since $f(x) \rightarrow 0$ as $x \rightarrow \infty$,

$$
\begin{aligned}
\mathbb{P}\left(T_{x}^{z} \geq(1+\varepsilon) \frac{z}{\mu}\right) & \leq \mathbb{P}\left(S_{(1+\varepsilon) z / \mu} \leq z-x+(1+\varepsilon) \frac{z}{\mu} f(x)\right) \\
& \leq \mathbb{P}\left(S_{(1+\varepsilon) z / \mu} \leq z\left(1+\frac{1}{2} \varepsilon\right)-x\right)
\end{aligned}
$$

provided only that $x$ is sufficiently large. Now the right-hand term converges to 0 , so it follows that

$$
\mathbb{P}\left(T_{x}^{z} \geq(1+\varepsilon) \frac{z}{\mu}\right) \rightarrow 0 .
$$

Note that we proved this result only for sufficiently large $x$, depending on $\varepsilon$. However, this restriction can be omitted, since, for fixed $x_{1}<x_{2}$, the random variables $T_{x_{1}}^{z}-T_{x_{2}}^{z}$ are bounded in probability uniformly in $z$. Thus, altogether we have, for any $x$,

$$
\mathbb{P}\left((1-\varepsilon) \frac{z}{\mu} \leq T_{x}^{z}<(1+\varepsilon) \frac{z}{\mu}\right) \rightarrow 1 \quad \text { as } z \rightarrow \infty,
$$

which (since $\varepsilon>0$ is arbitrary) is our assertion. 
We turn now to a central limit theorem for passage times of the processes $Y^{z}$. Choose $\kappa$ sufficiently large that $\sup _{y \geq \kappa} f(y) \leq \frac{1}{2} \mu$, and define the function $\beta_{z}, z \geq \kappa$, by

$$
\beta_{z}:=\int_{\kappa}^{z} \frac{\mathrm{d} y}{\mu-f(y)} .
$$

Proposition 3. Let

$$
\sigma^{2}:=\int_{(0, \infty)} y^{2} \lambda(\mathrm{d} y)<\infty
$$

Then, as $z \rightarrow \infty$,

$$
\frac{T_{x}^{z}-\beta_{z}}{\sqrt{z}} \stackrel{\mathrm{D}}{\rightarrow} N\left(0, \frac{\sigma^{2}}{\mu^{3}}\right) .
$$

Proof. (i) Note again that, for $x_{1}<x_{2}$, the random variables $T_{x_{1}}^{z}-T_{x_{2}}^{z}$ are bounded in probability uniformly in $z$. Thus, it suffices to prove our theorem for all $x \geq x_{0}$ for some $x_{0} \in \mathbb{R}$. Therefore, we may change $f(x)$ for all $x<x_{0}$; we do so in such a way that $f(x) \leq \frac{1}{2} \mu$ for all $x \in \mathbb{R}$, without touching the other properties of $f$. Thus, we assume from now that

$$
f(y) \leq \frac{1}{2} \mu \text { for all } y \in \mathbb{R},
$$

and set $\kappa=0$ in (11). Consequently,

$$
\frac{z}{\mu} \leq \beta_{z} \leq \frac{2 z}{\mu}, \quad z>0 .
$$

For any $z>0$, define the function $\rho^{z}(t)=\rho_{t}^{z}, 0 \leq t \leq \beta_{z}$, such that

$$
\beta_{\rho^{z}(t)}=\beta_{z}-t \text { for } 0 \leq t \leq \beta_{z},
$$

in particular, $\rho^{z}(0)=z$ and $\rho^{z}\left(\beta_{z}\right)=0$. This means that $\rho^{z}$ arises by first inverting the function $\beta$ (restricted to the interval $[0, z]$ ), and then reversing the time parameter on its domain $\left[0, \beta_{z}\right]$. Differentiation yields $\dot{\rho}_{t}^{z}=f\left(\rho_{t}^{z}\right)-\mu$, so $\dot{\rho}_{t} \leq-\frac{1}{2} \mu$ and

$$
\rho_{t}^{z}=z-\mu t+\int_{0}^{t} f\left(\rho_{s}^{z}\right) \mathrm{d} s .
$$

(ii) Inspection of (8) suggests that $\rho^{z}$ may be a good approximation for the process $Y^{z}$; we estimate the difference by observing that

$$
Y_{t}^{z}-\rho_{t}^{z}=-\left(S_{t}-\mu t\right)+\int_{0}^{t}\left(f\left(Y_{s}^{z}\right)-f\left(\rho_{s}^{z}\right)\right) \mathrm{d} s .
$$

For given $t>0$, define

$$
u_{t}= \begin{cases}\sup \left\{s<t: Y_{s}^{z} \leq \rho_{s}^{z}\right\} & \text { on the event } Y_{t}^{z}>\rho_{t}^{z}, \\ \sup \left\{s<t: Y_{s}^{z} \geq \rho_{s}^{z}\right\} & \text { on the event } Y_{t}^{z}<\rho_{t}^{z},\end{cases}
$$

and $u_{t}:=t$ on the event $Y_{t}^{z}=\rho_{t}^{z}$. Then $0 \leq u_{t} \leq t$ since $Y_{0}^{z}=z=\rho_{0}^{z}$. Because $f$ is a decreasing function, the event $Y_{t}^{z}>\rho_{t}^{z}$ implies that

$$
\begin{aligned}
Y_{t}^{z}-\rho_{t}^{z} & \leq Y_{t}^{z}-\rho_{t}^{z}-\int_{u_{t}}^{t}\left(f\left(Y_{s}^{z}\right)-f\left(\rho_{s}^{z}\right)\right) \mathrm{d} s-\left(Y_{u_{t}-}^{z}-\rho_{u_{t}-}^{z}\right) \\
& =-\left(S_{t}-\mu t\right)+\left(S_{u_{t}-}-\mu u_{t}\right) .
\end{aligned}
$$


On the event $Y_{t}^{z}<\rho_{t}^{z}$, there is an analogous estimate from below, so taken all together,

$$
\left|Y_{t}^{z}-\rho_{t}^{z}\right| \leq 2 M_{t}, \quad \text { where } \quad M_{t}:=\sup _{u \leq t}\left|S_{u}-\mu u\right| .
$$

Consequently, $Y_{s}^{z} \geq \rho_{s}^{z}-2 M_{s} \geq \rho_{s}^{z}-2 M_{t}$ for $s \leq t$ and from the monotonicity of $f$,

$$
\int_{0}^{t} f\left(Y_{s}^{z}\right) \mathrm{d} s-\int_{0}^{t} f\left(\rho_{s}^{z}\right) \mathrm{d} s \leq \int_{0}^{t} f\left(\rho_{s}^{z}-2 M_{t}\right) \mathrm{d} s-\int_{0}^{t} f\left(\rho_{s}^{z}\right) \mathrm{d} s \leq 2 M_{t} f\left(\rho_{t}^{z}-2 M_{t}\right) .
$$

An analoguous estimate is valid from below; it yields

$$
\left|\int_{0}^{t} f\left(Y_{s}^{z}\right) \mathrm{d} s-\int_{0}^{t} f\left(\rho_{s}^{z}\right) \mathrm{d} s\right| \leq 2 M_{t} f\left(\rho_{t}^{z}-2 M_{t}\right) .
$$

Recall here that, under our assumptions on the subordinator $S$, Donsker's invariance principle implies that

$$
M_{t}=O_{P}(\sqrt{t}) \quad \text { as } t \rightarrow \infty .
$$

(iii) Next we derive some upper estimates of probabilities. Given $a, x \in \mathbb{R}$, for any $c>0$,

$$
\begin{aligned}
& \mathbb{P}\left(T_{x}^{z} \geq \beta_{z}+a \sqrt{z}\right) \\
& =\mathbb{P}\left(Y_{t}^{z} \geq x \text { for all } t \leq \beta_{z}+a \sqrt{z}\right) \\
& =\mathbb{P}\left(S_{\beta_{z}+a \sqrt{z}} \leq z-x+\int_{0}^{\beta_{z}+a \sqrt{z}} f\left(Y_{s}^{z}\right) \mathrm{d} s, Y_{t}^{z} \geq x \text { for all } t \leq \beta_{z}+a \sqrt{z}\right) \\
& \leq \mathbb{P}\left(S_{\beta_{z}+a \sqrt{z}} \leq z-x+f(x)(c+|a|) \sqrt{z}+\int_{0}^{\beta_{z}-c \sqrt{z}} f\left(Y_{s}^{z}\right) \mathrm{d} s\right) .
\end{aligned}
$$

We now use (15). From the definition of $\rho^{z}$ and writing $\beta(y)=\beta_{y}$, we have

$$
\beta\left(\rho^{z}\left(\beta_{z}-c \sqrt{z}\right)\right)=c \sqrt{z}
$$

and then because of (14),

$$
\rho^{z}\left(\beta_{z}-c \sqrt{z}\right) \geq \frac{c \sqrt{z}}{2 \mu}
$$

So on the event $M_{\beta_{z}} \leq c \sqrt{z} /(8 \mu)$,

$$
\rho^{z}\left(\beta_{z}-\sqrt{z}\right)-2 M_{\beta_{z}-c \sqrt{z}} \geq \frac{c \sqrt{z}}{2 \mu}-\frac{c \sqrt{z}}{4 \mu}=\frac{c \sqrt{z}}{4 \mu} .
$$

Consequently, appealing to (15) and since $\beta_{z} \leq 2 z / \mu$,

$$
\begin{aligned}
\mathbb{P}\left(T_{x}^{z} \geq\right. & \left.\beta_{z}+a \sqrt{z}\right) \\
\leq & \mathbb{P}\left(M_{2 z / \mu}>\frac{c \sqrt{z}}{8 \mu}\right) \\
& +\mathbb{P}\left(S_{\beta_{z}+a \sqrt{z}} \leq z-x+f(x)(c+|a|) \sqrt{z}+\int_{0}^{\beta_{z}} f\left(\rho_{s}^{z}\right) \mathrm{d} s+\frac{c \sqrt{z}}{4 \mu} f\left(\frac{c \sqrt{z}}{4 \mu}\right)\right) .
\end{aligned}
$$


Furthermore, from the definition of $\rho^{z}$,

$$
z+\int_{0}^{\beta_{z}} f\left(\rho_{s}^{z}\right) \mathrm{d} s=\rho^{z}\left(\beta_{z}\right)+\mu \beta_{z}=\mu \beta_{z} .
$$

Therefore, if we fix $\varepsilon>0$, take $c$ so large that the first right-hand probability in (16) is smaller than $\varepsilon$, and then choose $z$ so large that $(c / 4 \mu) f(c \sqrt{z} / 4 \mu) \leq \varepsilon$ and also choose $x>0$ and so large that $c f(x)(c+|a|) \leq \varepsilon$, then

$$
\mathbb{P}\left(T_{x}^{z} \geq \beta_{z}+a \sqrt{z}\right) \leq \varepsilon+\mathbb{P}\left(S_{\beta_{z}+a \sqrt{z}} \leq \mu \beta_{z}+2 \varepsilon \sqrt{z}\right)
$$

Also, by the law of large numbers

$$
S_{\beta_{z}+a \sqrt{z}}-S_{\beta_{z}} \sim \mu a \sqrt{z} \quad \text { in probability. }
$$

Therefore,

$$
\mathbb{P}\left(T_{x}^{z} \geq \beta_{z}+a \sqrt{z}\right) \leq 2 \varepsilon+\mathbb{P}\left(S_{\beta_{z}} \leq \mu \beta_{z}+(-\mu a+3 \varepsilon) \sqrt{z}\right) .
$$

Also, $\mu \beta_{z} \sim z$, so, for large $z$,

$$
\mathbb{P}\left(T_{x}^{z} \geq \beta_{z}+a \sqrt{z}\right) \leq 2 \varepsilon+\mathbb{P}\left(S_{\beta_{z}} \leq \mu \beta_{z}+(-\mu a+4 \varepsilon) \mu^{1 / 2} \sqrt{\beta_{z}}\right) .
$$

It now follows from (12) and the central limit theorem that

$$
\frac{S_{t}-\mu t}{\sqrt{\sigma^{2} t}} \stackrel{\mathrm{D}}{\rightarrow} L
$$

where $L$ denotes a standard normal random variable. Thus,

$$
\limsup _{z \rightarrow \infty} \mathbb{P}\left(T_{x}^{z} \geq \beta_{z}+a \sqrt{z}\right) \leq 2 \varepsilon+\mathbb{P}\left(L \leq(-\mu a+4 \varepsilon) \mu^{1 / 2} \sigma^{-1}\right) .
$$

Note in our proof that the choice of $x$ depends on $\varepsilon$, but, since the differences $T_{x_{1}}^{z}-T_{x_{2}}^{z}$ are bounded in probability uniformly in $z$, this estimate generalizes to all $x$. Now letting $\varepsilon \rightarrow 0$ gives

$$
\limsup _{z \rightarrow \infty} \mathbb{P}\left(\frac{T_{x}^{z}-\beta_{z}}{\sqrt{z}} \geq a\right) \leq \mathbb{P}\left(L \leq-\frac{\mu^{3 / 2} a}{\sigma}\right) .
$$

This is the first part of our claim.

(iv) For the lower estimates, we first introduce the random variable

$$
R_{z, x}:=\sup \left\{t \geq 0: Y_{t}^{z} \geq x\right\}-\inf \left\{t \geq 0: Y_{t}^{z}<x\right\}
$$

this is the length of the time interval on which $Y_{t}^{z}-x$ changes from a positive sign to ultimately a negative sign (note that the paths of $Y^{z}$ are not monotone). We claim that these random variables are bounded in probability, uniformly in $z$ and $x$. Indeed, with

$$
\eta_{z, x}:=\inf \left\{t \geq 0: Y_{t}^{z}<x\right\},
$$

we have, for $t>\eta=\eta_{z, x}$, because of $Y_{\eta}^{z} \leq x$ and (13),

$$
Y_{t}^{z}=Y_{\eta}^{z}-\left(S_{t}-S_{\eta}\right)+\int_{\eta}^{t} f\left(Y_{s}^{z}\right) \mathrm{d} s \leq x-\left(S_{t}-S_{\eta}\right)+\frac{1}{2} \mu(t-\eta) .
$$


Thus, $R_{z, x}$ is bounded from above by

$$
R_{z, x}^{\prime}:=\sup \left\{u \geq 0:\left(S_{\eta_{z, x}+u}-S_{\eta_{z, x}}\right)-\frac{1}{2} \mu u \leq 0\right\} .
$$

These random variables are a.s. finite. Moreover, they are identically distributed, since the $\eta_{z, x}$ are stopping times. This proves that the $R_{z, x}$ are uniformly bounded in probability.

For the lower bounds and $a, b \in \mathbb{R}$, we have

$$
\begin{aligned}
\mathbb{P}\left(T_{x}^{z} \geq \beta_{z}+a \sqrt{z}\right) & \geq \mathbb{P}\left(Y_{t}^{z} \geq x \text { for all } t \leq \beta_{z}+a \sqrt{z}, R_{z, x} \leq b\right) \\
& =\mathbb{P}\left(Y_{t}^{z} \geq x \text { for all } \beta_{z}+a \sqrt{z}-b \leq t \leq \beta_{z}+a \sqrt{z}, R_{z, x} \leq b\right) .
\end{aligned}
$$

For these $t$,

$$
Y_{t}^{z}=z-S_{t}+\int_{0}^{t} f\left(Y_{s}^{z}\right) \mathrm{d} s \geq z-S_{\beta_{z}+a \sqrt{z}}+\int_{0}^{\beta_{z}+a \sqrt{z}-b} f\left(Y_{s}^{z}\right) \mathrm{d} s
$$

therefore,

$$
\begin{aligned}
\mathbb{P}\left(T_{x}^{z} \geq \beta_{z}+a \sqrt{z}\right) & \geq \mathbb{P}\left(S_{\beta_{z}+a \sqrt{z}} \leq z-x+\int_{0}^{\beta_{z}+a \sqrt{z}-b} f\left(Y_{s}^{z}\right) \mathrm{d} s, R_{z, x} \leq b\right) \\
& \geq \mathbb{P}\left(S_{\beta_{z}+a \sqrt{z}} \leq z-x+\int_{0}^{\beta_{z}-c \sqrt{z}} f\left(Y_{s}^{z}\right) \mathrm{d} s\right)-\mathbb{P}\left(R_{z, x}>b\right)
\end{aligned}
$$

for sufficiently large $c$.

We now use (15) as in (iii). Proceeding analogously, instead of estimate (16) we obtain

$$
\begin{aligned}
\mathbb{P}\left(T_{x}^{z} \geq \beta_{z}+a \sqrt{z}\right) \geq & -\mathbb{P}\left(R_{z, x}>b\right)-\mathbb{P}\left(M_{2 z / \mu}>\frac{c \sqrt{z}}{8 \mu}\right) \\
& +\mathbb{P}\left(S_{\beta_{z}+a \sqrt{z}} \leq z-x+\int_{0}^{\beta_{z}-c \sqrt{z}} f\left(\rho_{s}^{z}\right) \mathrm{d} s-\frac{c \sqrt{z}}{4 \mu} f\left(\frac{c \sqrt{z}}{4 \mu}\right)\right) .
\end{aligned}
$$

Also, since $\rho_{\beta_{z}}^{z}=0$ and $\dot{\rho}_{t}^{z} \leq-\frac{1}{2} \mu$,

$$
\int_{\beta_{z}-c \sqrt{z}}^{\beta_{z}} f\left(\rho_{s}^{z}\right) \mathrm{d} s \leq \int_{0}^{c \sqrt{z}} f\left(\frac{\mu s}{2}\right) \mathrm{d} s=o(\sqrt{z}) .
$$

Hence, for given $\varepsilon>0$ and sufficiently large $z$,

$$
\begin{aligned}
\mathbb{P}\left(T_{x}^{z} \geq \beta_{z}+a \sqrt{z}\right) \geq & -\mathbb{P}\left(R_{z, x}>b\right)-\mathbb{P}\left(M_{2 z / \mu}>\frac{c \sqrt{z}}{8 \mu}\right) \\
& +\mathbb{P}\left(S_{\beta_{z}+a \sqrt{z}} \leq z-\varepsilon \sqrt{z}+\int_{0}^{\beta_{z}} f\left(\rho_{s}^{z}\right) \mathrm{d} s-\frac{c \sqrt{z}}{4 \mu} f\left(\frac{c \sqrt{z}}{4 \mu}\right)\right) .
\end{aligned}
$$

Returning to the arguments of part (iii) we choose $b, c$ and then $z$ so large that

$$
\mathbb{P}\left(T_{x}^{z} \geq \beta_{z}+a \sqrt{z}\right) \geq-2 \varepsilon+\mathbb{P}\left(S_{\beta_{z}+a \sqrt{z}} \leq \mu \beta_{z}-2 \varepsilon \sqrt{z}\right)
$$

and

$$
\liminf _{z \rightarrow \infty} \mathbb{P}\left(T_{x}^{z} \geq \beta_{z}+a \sqrt{z}\right) \geq-3 \varepsilon+\mathbb{P}\left(L \leq-\frac{(\mu a+3 \varepsilon) \mu^{1 / 2}}{\sigma}\right) .
$$

The limit $\varepsilon \rightarrow 0$ leads to the desired lower estimate. 


\section{Approximating the block-counting process}

In this section we derive a strengthening of a result of Kersting et al. [4] on the approximation to the logarithm of the block-counting processes in the dust case. To this end, let us quickly recall the Poisson point process construction of the $\Lambda$-coalescent given in [4]; this is a slight variation of the construction provided by Pitman [6].

This construction requires $\Lambda(\{0\})=0$, a condition that is satisfied by coalescents with dust. Consider a Poisson point process $\Psi$ on $(0, \infty) \times(0,1] \times[0,1]^{n}$ with intensity

$$
\mathrm{d} t \times p^{-2} \Lambda(\mathrm{d} p) \times \mathrm{d} u_{1} \times \cdots \times \mathrm{d} u_{n}
$$

and let $\Pi_{n}(0)=\{\{1\}, \ldots,\{n\}\}$ be the partition of the set $\{1, \ldots, n\}$ into singletons. Suppose that $\left(t, p, u_{1}, \ldots, u_{n}\right)$ is a point of $\Psi$, and that $\Pi_{n}(t-)$ consists of the blocks $B_{1}, \ldots, B_{b}$, ranked in order by their smallest element. Then $\Pi_{n}(t)$ is obtained from $\Pi_{n}(t-)$ by merging together all of the blocks $B_{i}$ for which $u_{i} \leq p$ into a single block. These are the only times that mergers occur. This construction is well defined because, a.s. for any fixed $t^{\prime}<\infty$, there are only finitely many points $\left(t, p, u_{1}, \ldots, u_{n}\right)$ of $\Psi$ for which $t \leq t^{\prime}$ and at least two of $u_{1}, \ldots, u_{n}$ are less than or equal to $p$. The resulting process $\Pi_{n}=\left(\Pi_{n}(t), t \geq 0\right)$ is the $\Lambda$-coalescent. When $\left(t, p, u_{1}, \ldots, u_{n}\right)$ is a point of $\Psi$, we say that a $p$-merger occurs at time $t$.

Condition (7) allows us to approximate the number of blocks in the $\Lambda$-coalescent by a subordinator. Let $\phi:(0, \infty) \times(0,1] \times[0,1]^{n} \rightarrow(0, \infty) \times(0, \infty]$ be the function defined by

$$
\phi\left(t, p, u_{1}, \ldots, u_{n}\right)=(t,-\log (1-p)) .
$$

Now $\phi(\Psi)$ is a Poisson point process, and we can define a pure-jump subordinator $(S(t), t \geq 0)$ with the property that $S(0)=0$ and if $(t, x)$ is a point of $\phi(\Psi)$ then $S(t)=S(t-)+x$. With $\lambda$ the Lévy measure of $S,(9)$ and (12) now read

$$
\mu=\int_{[0,1]}|\log (1-p)| \frac{\Lambda(\mathrm{d} p)}{p^{2}} \quad \text { and } \quad \sigma^{2}=\int_{[0,1]}(\log (1-p))^{2} \frac{\Lambda(\mathrm{d} p)}{p^{2}} .
$$

This subordinator first appeared in [6] and was used to approximate the block-counting process by Gnedin et al. [1] and Möhle [5]; the benefits of a refined approximation by a drifted subordinator were discovered in [4]. Recall that the drift appears because a merging of $b$ out of $N_{n}(t)$ lines results in a decrease of $b-1$ and not of $b$ lines; see [4, Equation (23)] for an explanation of the form of the drift. Our next result provides a refinement of [4, Theorem 10].

Proposition 4. Suppose that $\int_{[0,1]} p^{-1} \Lambda(\mathrm{d} p)<\infty$. Let $f$ be as in (2), and let $Y_{n}$ be the solution of (8) with $z:=\log n$. Then, for any $\varepsilon>0$, there exists $\ell<\infty$ such that

$$
\mathbb{P}\left(\sup _{t<\tau_{n}}\left|\log N_{n}(t)-Y_{n}(t)\right| \leq \ell, Y_{n}\left(\tau_{n}\right)<\ell\right) \geq 1-\varepsilon .
$$

Proof. From [4] we know that, for given $\varepsilon>0$, there exists $r<\infty$ such that

$$
\mathbb{P}\left(\sup _{t<\tau_{n}}\left|\log N_{n}(t)-Y_{n}(t)\right| \leq r\right) \geq 1-\frac{1}{2} \varepsilon .
$$

Consider the size $\Delta_{n}$ of the last jump. Letting $\left(u_{i}, p_{i}\right), i \geq 1$, be the points of the underlying Poisson point process with intensity measure $\mathrm{d} t \Lambda(\mathrm{d} p) / p^{2}$, the associated subordinator $S$ has 
jumps of size $v_{i}=-\log \left(1-p_{i}\right)$ at times $t_{i}$. So, for any $c>0$, the event $\left\{\Delta_{n} \leq \log N_{n}\left(\tau_{n}-\right)-c\right\}$ is the same as

$$
\begin{gathered}
\left\{\tau_{n}=t_{i} \text { and }-\log \left(1-p_{i}\right) \leq \log N_{n}\left(t_{i}-\right)-c \text { for some } i \geq 1\right\} \\
\quad=\left\{\tau_{n}=t_{i} \text { and } p_{i} \leq 1-\frac{\mathrm{e}^{c}}{N_{n}\left(t_{i}-\right)} \text { for some } i \geq 1\right\}
\end{gathered}
$$

Given $N_{n}(t-)$, this event appears at time $t$ at rate

$$
v_{n, t}=\int_{\left[0,1-\mathrm{e}^{c} / N_{n}(t-)\right]} p^{N_{n}(t-)} \frac{\Lambda(\mathrm{d} p)}{p^{2}} .
$$

Using the inequalities $p^{b}=(1-(1-p))^{b} \leq \mathrm{e}^{-(1-p) b} \leq 1 /((1-p) b)$, we obtain

$$
v_{n, t} \leq \int_{\left[0,1-\mathrm{e}^{c} / N_{n}(t-)\right]} \mathrm{e}^{-(1-p)\left(N_{n}(t-)-2\right)} \Lambda(\mathrm{d} p) \leq \int_{\left[0,1-\mathrm{e}^{c} / N_{n}(t-)\right]} \frac{\mathrm{e}^{2}}{(1-p) N_{n}(t-)} \Lambda(\mathrm{d} p) .
$$

It follows that

$$
\mathbb{E}\left[\int_{0}^{\infty} v_{n, t} \mathrm{~d} t\right] \leq \mathbb{E}\left[\int_{[0,1]} \int_{0}^{\infty} \frac{\mathrm{e}^{2}}{(1-p) N_{n}(t-)} \mathbf{1}_{\left\{N_{n}(t-) \geq\left\lceil\mathrm{e}^{c} /(1-p)\right\rceil\right\}} \mathrm{d} t \Lambda(\mathrm{d} p)\right] .
$$

Lemma 14 of [4] yields the estimate

$$
\mathbb{E}\left[\int_{0}^{\infty} \frac{1}{N_{n}(t-)} \mathbf{1}_{\left\{N_{n}(t-) \geq\left\lceil\mathrm{e}^{c} /(1-p)\right\rceil\right\}} \mathrm{d} t\right] \leq c_{1}\left\lceil\frac{\mathrm{e}^{c}}{1-p}\right\rceil^{-1} \leq c_{1} \frac{1-p}{\mathrm{e}^{c}}
$$

for some $c_{1}>0$; hence,

$$
\mathbb{E}\left[\int_{0}^{\infty} v_{n, t} \mathrm{~d} t\right] \leq c_{1} \mathrm{e}^{2-c} \Lambda([0,1])
$$

Therefore, for sufficiently large $c$,

$$
\mathbb{E}\left[\int_{0}^{\infty} v_{n, t} \mathrm{~d} t\right] \leq \frac{1}{2} \varepsilon
$$

implying that

$$
\mathbb{P}\left(\Delta_{n} \leq \log N_{n}\left(\tau_{n}-\right)-c\right)=1-\exp \left(-\mathbb{E}\left[\int_{0}^{\infty} v_{n, t} \mathrm{~d} t\right]\right) \leq \frac{1}{2} \varepsilon .
$$

Altogether we obtain

$$
\mathbb{P}\left(\sup _{t<\tau_{n}}\left|\log N_{n}(t)-Y_{n}(t)\right| \leq r, \Delta_{n}>\log N_{n}\left(\tau_{n}-\right)-c\right) \geq 1-\varepsilon .
$$

The event in the last relation implies that

$$
Y_{n}\left(\tau_{n}\right)=Y_{n}\left(\tau_{n}-\right)-\Delta_{n}<\log N_{n}\left(\tau_{n}-\right)+r-\left(\log N_{n}\left(\tau_{n}-\right)-c\right)=r+c,
$$

and the claim of the proposition follows with $\ell=r+c$. 


\section{Proof of the main results}

Proof of Theorem 1. Assume first that $\mu<\infty$. Then we have a coalescent with dust, and we can apply Proposition 4. Fix $\eta>0$. Note that, on the event that $Y_{n}\left(\tau_{n}\right)<\ell$, the event $\tau_{n}<(1-\eta) \log n / \mu$ implies the inequality $T_{\ell}^{\log n}<(1-\eta) \log n / \mu$, where $T_{x}^{z}$ is defined following (8). Thus, in view of Proposition 4 , for any $\varepsilon>0$, there exists $\ell$ such that

$$
\mathbb{P}\left(\tau_{n}<\frac{(1-\eta) \log n}{\mu}\right) \leq \mathbb{P}\left(T_{\ell}^{\log n}<\frac{(1-\eta) \log n}{\mu}\right)+\varepsilon .
$$

Proposition 2 implies that the right-hand probability converges to 0 as $n \rightarrow \infty$. Letting $\varepsilon \rightarrow 0$ we obtain

$$
\lim _{n \rightarrow \infty} \mathbb{P}\left(\tau_{n}<\frac{(1-\eta) \log n}{\mu}\right)=0 .
$$

Also, on the event $\sup _{t<\tau_{n}}\left|\log N_{n}(t)-Y_{n}(t)\right| \leq \ell$, the event $\tau_{n}>(1+\eta) \log n / \mu$ implies that $Y_{n}(t) \geq-\ell$ for all $t \leq(1+\eta) \log n / \mu$, and, consequently,

$$
\mathbb{P}\left(\tau_{n}>\frac{(1+\eta) \log n}{\mu}\right) \leq \mathbb{P}\left(T_{-\ell}^{\log n}>\frac{(1+\eta) \log n}{\mu}\right)+\varepsilon .
$$

Again, from Proposition 2, the right-hand probability converges to 0, and we obtain

$$
\lim _{n \rightarrow \infty} \mathbb{P}\left(\tau_{n}>\frac{(1+\eta) \log n}{\mu}\right)=0 .
$$

Thus, our claim follows in the $\mu<\infty$ case.

Now assume that $\mu=\infty$. If $\Lambda(\{0\})>0$ then the coalescent comes down from $\infty$ and $\tau_{n}$ remains bounded in probability. The same is true if $\Lambda(\{1\})>0$; thus, we may assume that $\Lambda(\{0,1\})=0$.

For given $\varepsilon>0$, define the measure $\Lambda^{\varepsilon}$ by $\Lambda^{\varepsilon}(B):=\Lambda(B \cap[\varepsilon, 1-\varepsilon])$. Obviously,

$$
\mu^{\varepsilon}:=\int_{0}^{1}|\log (1-p)| \frac{\Lambda^{\varepsilon}(\mathrm{d} p)}{p^{2}}<\infty .
$$

Thus, for the absorption times $\tau_{n}^{\varepsilon}$ of the $\Lambda^{\varepsilon}$-coalescent, we have

$$
\frac{\tau_{n}^{\varepsilon}}{\log n} \rightarrow \frac{1}{\mu^{\varepsilon}}
$$

in probability as $n \rightarrow \infty$. Now we may couple the $\Lambda^{\varepsilon}$-coalescent in an obvious manner to the $\Lambda$-coalescent in such a way that $N_{n}(t) \leq N_{n}^{\varepsilon}(t)$ a.s. for all $t \geq 0$, in particular $\tau_{n} \leq \tau_{n}^{\varepsilon}$. Hence, it follows that

$$
\mathbb{P}\left(\frac{\tau_{n}}{\log n}>\frac{2}{\mu^{\varepsilon}}\right) \rightarrow 0 .
$$

Because $\Lambda(\{0,1\})=0, \mu^{\varepsilon} \rightarrow \mu=\infty$ as $\varepsilon \rightarrow 0$, and, consequently,

$$
\mathbb{P}\left(\frac{\tau_{n}}{\log n}>\eta\right) \rightarrow 0
$$

for all $\eta>0$. This is our claim. 
Proof of Theorem 2. Because of the condition $\mu<\infty$ we may again apply Proposition 4; we follow the same line as in the previous proof. For $\varepsilon>0$, there exists $\ell$ such that, for all $a \in \mathbb{R}$,

$$
\mathbb{P}\left(\tau_{n}<b_{n}+a \sqrt{n}\right) \leq \mathbb{P}\left(T_{\ell}^{\log n}<b_{n}+a \sqrt{n}\right)+\varepsilon
$$

and

$$
\mathbb{P}\left(\tau_{n}>b_{n}+a \sqrt{n}\right) \leq \mathbb{P}\left(T_{-\ell}^{\log n}>b_{n}+a \sqrt{n}\right)+\varepsilon .
$$

Now apply Proposition 3 and let $\varepsilon \rightarrow 0$.

Proof of Proposition 1. (i) Start by assuming that (6) holds. Because $1-(1-p)^{1 / r} \leq$ $\min (p / r, 1)$ for $0<r<1$, we have, for $\alpha>0$,

$$
f\left(\log \frac{1}{r}\right) \leq \int_{0}^{r^{\alpha}} \frac{\Lambda(\mathrm{d} p)}{p}+r \int_{r^{\alpha}}^{1} \frac{\Lambda(\mathrm{d} p)}{p^{2}} \leq \int_{0}^{r^{\alpha}} \frac{\Lambda(\mathrm{d} p)}{p}+r^{1-\alpha} \int_{0}^{1} \frac{\Lambda(\mathrm{d} p)}{p} .
$$

Also, since $1-(1-p)^{1 / r} \geq 1-\mathrm{e}^{-p / r} \geq \mathrm{e}^{-p / r} p / r$, it follows that, for $\beta>0$,

$$
f\left(\log \frac{1}{r}\right) \geq \mathrm{e}^{-r^{\beta-1}} \int_{0}^{r^{\beta}} \frac{\Lambda(\mathrm{d} p)}{p} .
$$

Together with (6), these two estimates imply that, for $\alpha<1<\beta$,

$$
c \beta^{-1 / 2} \leq \liminf _{r \rightarrow 0} f\left(\log \frac{1}{r}\right) \sqrt{\log \frac{1}{r}} \leq \limsup _{r \rightarrow 0} f\left(\log \frac{1}{r}\right) \sqrt{\log \frac{1}{r}} \leq c \alpha^{-1 / 2} .
$$

Letting $\alpha, \beta \rightarrow 1$ we arrive at $f(y)=(c+o(1)) / \sqrt{y}$ as $y \rightarrow \infty$ and, consequently,

$$
\int_{0}^{\log n} f(y) \mathrm{d} y=(c+o(1)) 2 \sqrt{\log n} \quad \text { as } n \rightarrow \infty .
$$

Now, since

$$
\frac{1}{\mu-f(y)}=\frac{1}{\mu}+\frac{f(y)}{\mu(\mu-f(y))}
$$

and $f(y)=o(1)$ as $y \rightarrow \infty$,

$$
\int_{\kappa}^{z} \frac{\mathrm{d} y}{\mu-f(y)}=\frac{z}{\mu}+\frac{1+o(1)}{\mu^{2}} \int_{0}^{z} f(y) \mathrm{d} y+O(1) \quad \text { as } z \rightarrow \infty
$$

so, as claimed,

$$
b_{n}=\frac{\log n}{\mu}+\frac{2 c+o(1)}{\mu^{2}} \sqrt{\log n} .
$$

(ii) Suppose now that (5) is satisfied. Then, from (19) with $z=\log n$, it follows that

$$
\int_{0}^{\log n} f(y) \mathrm{d} y=(2 c+o(1)) \sqrt{\log n} \quad \text { as } n \rightarrow \infty,
$$

or, equivalently,

$$
\int_{0}^{z} f(y) \mathrm{d} y=(2 c+o(1)) \sqrt{z} \quad \text { as } z \rightarrow \infty .
$$

This implies that $f(z)=(c+o(1)) / \sqrt{z}$ as $z \rightarrow \infty$. For $c=0$, this claim follows because $f$ is decreasing; hence,

$$
z f(z) \leq \int_{0}^{z} f(y) \mathrm{d} y=o(\sqrt{z})
$$


For $c>0$, we use the estimate

$$
\frac{1}{\eta \sqrt{z}} \int_{z}^{(1+\eta) z} f(y) \mathrm{d} y \leq \sqrt{z} f(z) \leq \frac{1}{\eta \sqrt{z}} \int_{(1-\eta) z}^{z} f(y) \mathrm{d} y
$$

with $\eta>0$. Taking the limit $z \rightarrow \infty$ and then $\eta \rightarrow 0$ yields $f(z)=(c+o(1)) / \sqrt{z}$. Now, similarly to part (i), from (17) and (18), we obtain

$$
c \sqrt{\alpha} \leq \liminf _{r \rightarrow 0} \sqrt{\log \frac{1}{r}} \int_{[0, r]} \frac{\Lambda(\mathrm{d} p)}{p} \leq \limsup _{r \rightarrow 0} \sqrt{\log \frac{1}{r}} \int_{[0, r]} \frac{\Lambda(\mathrm{d} p)}{p} \leq c \sqrt{\beta} .
$$

Letting $\alpha, \beta \rightarrow 1$ we arrive at (6).

\section{Acknowledgement}

It is our pleasure to dedicate this work to Peter Jagers.

\section{References}

[1] Gnedin, A., Iksanov, A., And Marynych, A. (2011). On $\Lambda$-coalescents with dust component. J. Appl. Prob. 48, $1133-1151$.

[2] Goldschmidt, C. and Martin, J. B. (2005). Random recursive trees and the Bolthausen-Sznitman coalesent. Electron. J. Prob. 10, 718-745.

[3] Herriger, P. ANd MöHLe, M. (2012). Conditions for excheangable coalescents to come down from infinity. ALEA 9, 637-665.

[4] Kersting, G., Schweinsberg, J., And Wakolbinger, A. (2018). The size of the last merger and time reversal in $\Lambda$-coalescents. Ann. Inst. H. Poincaré Prob. Statist. 54, 1527-1555.

[5] MöHLE, M. (2014). On hitting probabilities of beta coalescents and absorption times of coalescents that come down from infinity. ALEA 11, 141-159.

[6] Pitman, J. (1999). Coalescents with multiple collisions. Ann. Prob. 27, 1870-1902.

[7] Sagitov, S. (1999). The general coalescent with asynchronous mergers of ancestral lines. J. Appl. Prob. 36, 1116-1125.

[8] Schweinsberg, J. (2000). A necessary and sufficient condition for the $\Lambda$-coalescent to come down from infinity. Electron. Commun. Prob. 5, 1-11.

\section{GÖTZ KERSTING, Goethe University Frankfurt}

Institute of Mathematics, Goethe University Frankfurt, Robert Mayer Strasse 10, 60325 Frankfurt am Main, Germany. Email address: kersting@math.uni-frankfurt.de

\section{ANTON WAKOLBINGER, Goethe University Frankfurt}

Institute of Mathematics, Goethe University Frankfurt, Robert Mayer Strasse 10, 60325 Frankfurt am Main, Germany. Email address: wakolbinger@math.uni-frankfurt.de 